\title{
Error-Disturbance Uncertainty Relations in Neutron-Spin Measurements
}

Stephan Sponar, Georg Sulyok, Jaqueline Erhart, and Yuji Hasegawa

Atominstitut, Vienna University of Technology, Stadionallee 2, 1020 Vienna, Austria

Correspondence should be addressed to Stephan Sponar; sponar@ati.ac.at

Received 2 June 2014; Accepted 28 July 2014; Published 8 September 2014

Academic Editor: Guillaume Pignol

Copyright (C) 2014 Stephan Sponar et al. This is an open access article distributed under the Creative Commons Attribution License, which permits unrestricted use, distribution, and reproduction in any medium, provided the original work is properly cited. The publication of this article was funded by SCOAP ${ }^{3}$.

In his seminal paper, which was published in 1927, Heisenberg originally introduced a relation between the precision of a measurement and the disturbance it induces onto another measurement. Here, we report a neutron-optical experiment that records the error of a spin-component measurement as well as the disturbance caused on a measurement of another spin-component to test error-disturbance uncertainty relations (EDRs). We demonstrate that Heisenberg's original EDR is violated and the Ozawa and Branciard EDRs are valid in a wide range of experimental parameters.

\section{Introduction}

The uncertainty principle represents, without any doubt, one of the most important cornerstones of the Copenhagen interpretation of quantum theory. In his celebrated paper from 1927 [1], Heisenberg gives at least two distinct statements about the limitations on preparation and measurement of physical systems: (i) incompatible observables cannot be measured with arbitrary accuracy: a measurement of one of these observables disturbs the other one accordingly, and vice versa; (ii) it is impossible to prepare a system such that a pair of noncommuting (incompatible) observables are arbitrarily well defined. In [1], the observables are represented by position and momentum.

In his original paper [1], Heisenberg proposed a reciprocal relation for measurement error and disturbance by the famous $\gamma$-ray microscope thought experiment: "At the instant when the position is determined-therefore, at the moment when the photon is scattered by the electron-the electron undergoes a discontinuous change in momentum. This change is the greater the smaller the wavelength of the light employedthat is, the more exact the determination of the position..." [1]. Heisenberg follows Einstein's realistic view, that is, to base a new physical theory only on observable quantities (elements of reality), arguing that terms like velocity or position make no sense without defining an appropriate apparatus for a measurement. By solely considering the Compton effect, Heisenberg gives a rather heuristic estimate for the product of the inaccuracy (error) of a position measurement $p_{1}$ and the disturbance $q_{1}$ induced on the particles momentum, denoted by

$$
p_{1} q_{1} \sim h .
$$

According to (1), it can be referred to as a measurement uncertainty (i) or as an error-disturbance uncertainty relation (EDR).

Heisenberg's original formulation $[1,2]$ can be read in modern treatment as $\epsilon(Q) \eta(P) \geq \hbar / 2$, for error $\epsilon(Q)$ of a measurement of the position observable $Q$ and disturbance $\eta(P)$ of the momentum observable $P$ induced by the position measurement. However, most modern textbooks introduce the uncertainty relation in terms of a preparation uncertainty (ii) relation denoted by

$$
\Delta(Q) \Delta(P) \geq \hbar / 2 .
$$

Equation (2) was proved by Kennard in 1927 [3] for the standard deviations $\Delta(Q)$ and $\Delta(P)$ of the position observable $Q$ and the momentum observable $P$, given by $\Delta(X)=$ $\sqrt{\left\langle X^{2}\right\rangle-\langle X\rangle^{2}}$. But this is a different physical situation: here 
statistical distributions of not a joint but a single measurement of either $Q$ or $P$ are considered. Kennard's relation addresses an intrinsic uncertainty which every quantum system must possess, independent of whether it is measured or not. The unavoidable recoil caused by the measuring device is ignored here. Later Robertson generalized Kennards relation between standard deviations to arbitrary pairs of observables $A$ and $B$ :

$$
\Delta(A) \Delta(B) \geq \frac{1}{2}|\langle\psi|[A, B]| \psi\rangle|
$$

Robertson's relation (3) has been confirmed by many different experiments [4-6] and is uncontroversial.

A corresponding generalized form of Heisenberg's original error-disturbance uncertainty relation would read

$$
\epsilon(A) \eta(B) \geq \frac{1}{2}|\langle\psi|[A, B]| \psi\rangle|
$$

However, certain measurements do not obey (4) [7-9], proving (4) to be formally incorrect.

In 2003, Ozawa introduced the correct form of a generalized error-disturbance uncertainty relation based on rigorous theoretical treatments of quantum measurements:

$$
\epsilon(A) \eta(B)+\epsilon(A) \Delta(B)+\Delta(A) \eta(B) \geq \frac{1}{2}|\langle\psi|[A, B]| \psi\rangle|,
$$

where $\epsilon(A)$ denotes the root-mean-square (r.m.s.) error of an arbitrary measurement for an observable $A, \eta(B)$ is the r.m.s. disturbance on another observable $B$ induced by the measurement, and $\Delta(A)$ and $\Delta(B)$ are the standard deviations of $A$ and $B$ in the state $|\psi\rangle$ before the measurement. Ozawa's inequality (5) was tested experimentally with neutronic $[10$, 11] and photonic [12-14] systems.

Though universally valid, Ozawa's relations (5) are not optimal. Recently, Branciard [15] has revised Ozawa's EDR, resulting in a tight $\mathrm{EDR}$, describing the now optimal tradeoff relation between error $\epsilon(A)$ and disturbance $\eta(B)$ :

$$
\begin{aligned}
& \epsilon(A)^{2} \Delta(B)^{2}+\Delta(A)^{2} \eta(B)^{2} \\
& \quad+2 \sqrt{\Delta(A)^{2} \Delta(B)^{2}-C_{A B}^{2}} \epsilon(A) \eta(B) \geq C_{A B}^{2},
\end{aligned}
$$

with $C_{A B}=1 / 2|\langle\psi|[A, B]| \psi\rangle|$. Experimental demonstrations of (6) using photons are reported in $[16,17]$.

\section{Materials and Methods}

In our experiment the error-disturbance uncertainty relations, as defined in (5) and (6), are tested via a successive measurement for spin observables $A$ and $B$. The experimental scheme is depicted in Figure 1. The observables $A$ and $B$ are set as the $x$ and $y$ components of the neutron $1 / 2$ spin. (For simplicity, $\hbar / 2$ is omitted for each spin component.) The error $\epsilon(A)$ and the disturbance $\eta(B)$ are defined for a joint measurement apparatus, so that apparatus A1 measures the observable $A=\sigma_{x}$ with error $\epsilon(A)$ and disturbs the

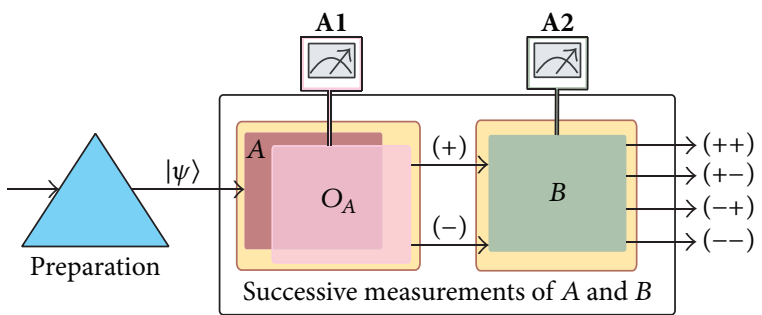

Figure 1: A successive measurement scheme of observables $A$ and $B$ exploited for the demonstration of the error-disturbance uncertainty relation. After state preparation (blue) apparatus A1 carries out a projective measurement of $O_{A}$ instead of $A$ (light red), thereby disturbing observable $B$ which is detected by apparatus A2 (green), error $\epsilon(A)$ and disturbance $\eta(B)$ are quantitatively determined by the four possible outcomes denoted by $(++),(+-),(-+)$, and $(--)$.

observable $B=\sigma_{y}$ thereby with disturbance $\eta(B)$ during the measurement (here $\sigma_{x}$ and $\sigma_{y}$ denote the Pauli matrices). Finally apparatus $\mathbf{A} 2$ measures $B=\sigma_{y}$. To control the error $\epsilon(A)$ and the disturbance $\eta(B)$, apparatus $\mathbf{A 1}$ is designed to actually carry out not the maximally disturbing projective measurement $A=\sigma_{x}$, but the projective measurement along a distinct axis $\vec{o}_{a}(\theta, \phi)$ denoted by $O_{A}=\vec{o}_{a}(\theta, \phi) \cdot \vec{\sigma}$, where $\vec{\sigma}=$ $\left(\sigma_{x}, \sigma_{y}, \sigma_{z}\right)^{T}$. Here $\theta, \phi$ denote polar and azimuthal angle of the measurement direction $\vec{o}_{a}$ and are experimentally controlled detuning parameters, so that $\epsilon(A)$ and $\eta(B)$ are determined as a function of $\phi$ and $\theta$. A schematic illustration of the experimental apparatus for successive neutron-spin measurements is given in Figure 2.

For (5) and (6), error $\epsilon(A)$ and disturbance $\eta(B)$ are defined via an indirect measurement model for an apparatus A measuring an observable $A$ of an object system $\mathbf{S}$ as

$$
\begin{gathered}
\epsilon(A)=\|\left(U^{\dagger}(\mathbb{1} \otimes M) U-A \otimes \mathbb{1}\right)|\psi\rangle|\xi\rangle \|, \\
\eta(B)=\|\left(U^{\dagger}(B \otimes \mathbb{1}) U-B \otimes \mathbb{1}\right)|\psi\rangle|\xi\rangle \|,
\end{gathered}
$$

where $|\psi\rangle$ is the state before the measurement of system $\mathbf{S}$, which is described by a Hilbert space $\mathscr{H}^{\text {obj }}$, and $|\xi\rangle$ and $M$ are the initial state of the probe system $\mathbf{P}$ (in Hilbert space $\mathscr{H}^{\text {pro }}$ ) and an observable $M$, referred to as meter observable, of $\mathbf{P}$ which accounts for the meter of the apparatus. A unitary operator $U$ on $\mathscr{H}^{\text {obj }} \otimes \mathscr{H}^{\text {pro }}$ describes the time evolution of the composite system $\mathbf{S}+\mathbf{P}$ during the measurement interaction. Here the Euclidean norm is used where the norm of a state vector in Hilbert space $X|\psi\rangle$ is given by the square root of its inner product: $\| X|\psi\rangle \|=\left\langle\psi\left|X^{\dagger} X\right| \psi\right\rangle^{1 / 2}$. A schematic illustration of a measurement apparatus $\mathbf{A}$ is given in Figure 3.

A nondegenerate meter observable $M$ has a spectral decomposition $M=\sum_{m} m|m\rangle\langle m|$, where $m$ varies over eigenvalues of $M$, and then the apparatus $\mathbf{A}$ has a family $\left\{M_{m}\right\}$ of operators, called the measurement operators, acting on $\mathscr{H}^{\text {obj }}$ and defined as $M_{m}=\langle m|U| \xi\rangle$. Hence, the error is given by $\epsilon(A)^{2}=\sum_{m} \| M_{m}(m-A)|\psi\rangle \|^{2}$. If $M_{m}$ are mutually orthogonal, projection operators sum and norm can be exchanged and the error can be written in compact form as 


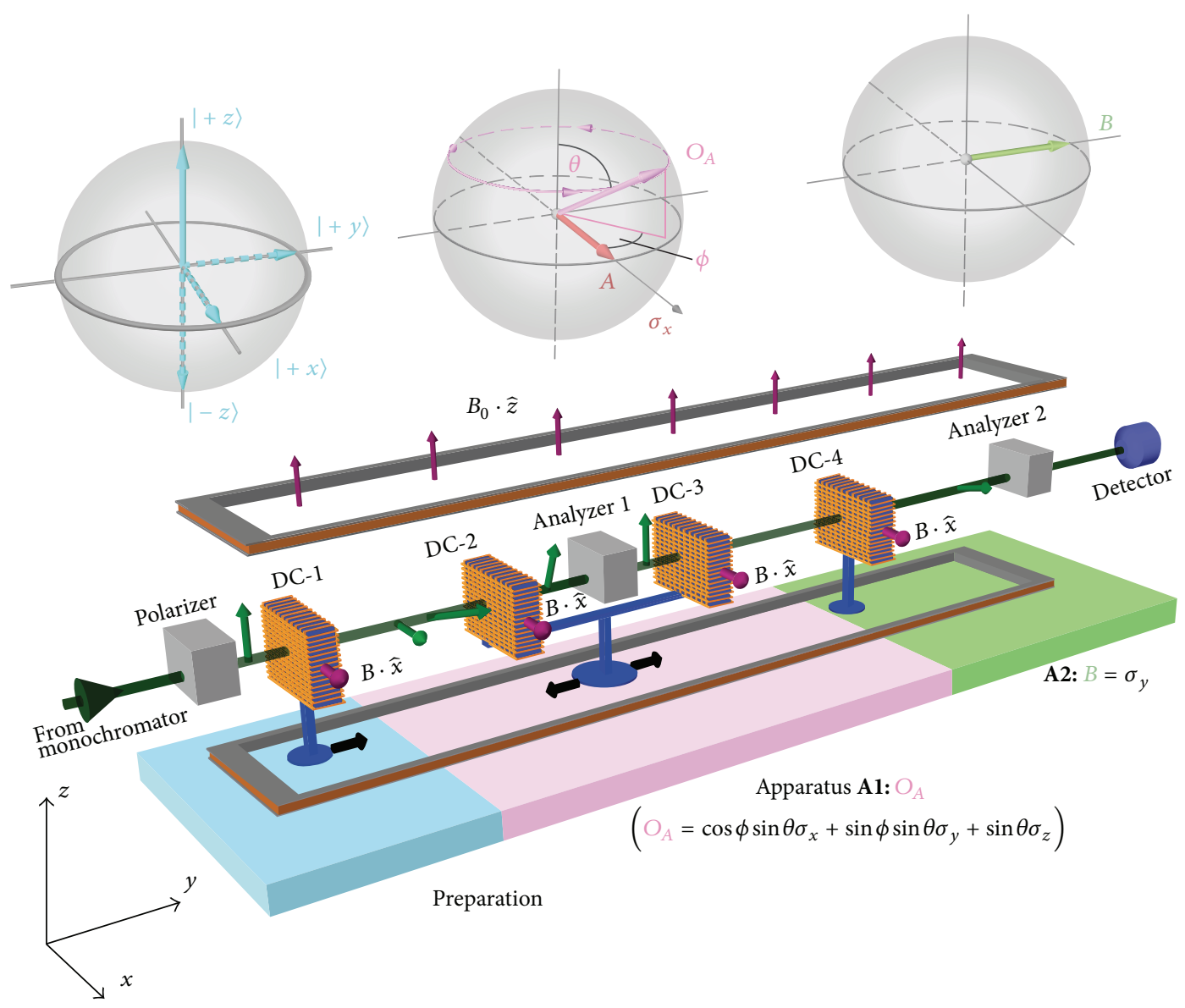

FIGURE 2: Neutron polarimetric setup for demonstration of the universally valid uncertainty relation for error and disturbance in neutronspin measurements. The setup is divided into three stages: state preparation (blue region), apparatus Al carrying out the measurement of observable $O_{A}=\vec{o}_{a}(\theta, \phi) \cdot \vec{\sigma}$ (red region), and apparatus A2 performing the measurement of observable $B=\sigma_{y}$ (green region). All required terms of (5), that is, error $\epsilon(A)$ and disturbance $\eta(B)$ as well as the standard deviations $\Delta(A)$ and $\Delta(B)$, are determined from the expectation values of the successive measurement.

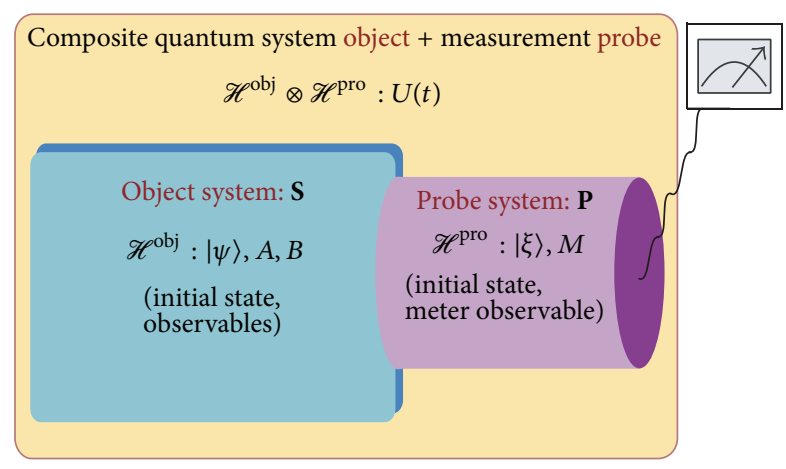

Figure 3: An indirect measurement model for apparatus A measuring an object system $\mathbf{S}$, defined on Hilbert space $\mathscr{H}^{\text {obj }}$, is specified by a quadruple $\left(\mathscr{H}^{\text {pro }},|\xi\rangle, U(t), M\right)$.

$\epsilon(A)^{2}=\sum_{m} \|\left(O_{A}-A\right)|\psi\rangle \|^{2}$, where $O_{A}$ is the output operator given as $O_{A}=\sum_{m} m M_{m}$. The disturbance can be written as $\eta(B)^{2}=\sum_{m} \|\left[M_{m}, B\right]|\psi\rangle \|^{2}$. All these calculations are elaborated in detail in [18].
In our experiment, the measuring apparatus $\mathbf{A 1}$ is considered to carry out a projective spin measurement along a distinct axis $\vec{o}_{a}(\theta, \phi)$ denoted by $O_{A}=\vec{o}_{a}(\theta, \phi) \cdot \vec{\sigma}=M_{+1}-M_{-1}$ (where $M_{ \pm 1}=1 / 2\left(\mathbb{1} \pm \vec{o}_{a}(\theta, \phi) \cdot \vec{\sigma}\right)$ ) instead of precisely $A=\sigma_{x}$. In order to detect the disturbance $\eta(B)$ on the observable $B$, induced by measuring $O_{A}$, apparatus A2 carries out the projective measurement of $B=\sigma_{y}$ in the state just after the first measurement. Though claimed to be experimentally inaccessible $[19,20]$, in the case of projection operators error $\epsilon(A)$ and disturbance $\eta(B)$ can be expressed as a sum of expectation values in three different states, applying the method proposed in [21]. Using the modified output operators of the apparatus $\mathbf{A} 2$ defined as $X_{B}=M_{+1} B M_{+1}+M_{-1} B M_{-1}$ and $X_{B}^{(2)}=M_{+1} B^{2} M_{+1}+M_{-1} B^{2} M_{-1}$, measurement error and disturbance are given by

$$
\begin{aligned}
\epsilon(A)^{2}= & +\left\langle\psi\left|O_{A}\right| \psi\right\rangle+\left\langle\psi\left|A O_{A} A\right| \psi\right\rangle \\
& -\left\langle\psi\left|(A+\mathbb{1}) O_{A}(A+\mathbb{1})\right| \psi\right\rangle, \\
\eta(B)^{2}= & 2+\left\langle\psi\left|X_{B}\right| \psi\right\rangle+\left\langle\psi\left|B X_{B} B\right| \psi\right\rangle \\
& -\left\langle\psi\left|(B+\mathbb{1}) X_{B}(B+\mathbb{1})\right| \psi\right\rangle .
\end{aligned}
$$


The expectation values of $O_{A}$ in a state $|\psi\rangle$ (see (8a) and (8b)), necessary for the determination of error $\epsilon(A)$, are derived from the intensities at the four possible output ports, depicted in Figure 4, denoted by $I_{++}, I_{+-}, I_{-+}$, and $I_{--}$. The expectation value is obtained from the following combination of count rates: $\left\langle\psi\left|O_{A}\right| \psi\right\rangle=\left(I_{++}+I_{+-}-I_{-+}-I_{--}\right) /\left(I_{++}+\right.$ $\left.I_{+-}+I_{-+}+I_{--}\right)$, using intensities at the four possible output ports, indicating which projections have been carried out. As already discussed due to the prior measurement of $O_{A}$, the operator of apparatus $\mathbf{A 2}$ is modified from $B$ to $X_{B}$, with the corresponding expectation value expressed as $\left\langle\psi\left|X_{B}\right| \psi\right\rangle=\left(I_{++}+I_{-+}-I_{+-}-I_{--}\right) /\left(I_{++}+I_{+-}+I_{-+}+I_{--}\right)$, required to determine the disturbance $\eta(B)$. Consequently all expectation values necessary to determine error $\epsilon(A)$ and disturbance $\eta(B)$ can be derived from the intensities in the three input states $|\psi\rangle, A|\psi\rangle,(A+\mathbb{1})|\psi\rangle$ and $|\psi\rangle, B|\psi\rangle,(B+$ $\mathbb{1})|\psi\rangle$, respectively.

These states are generated by spinor rotations within DC1 and induced by the guide field, due to an appropriate coil position within the preparation section (blue) of the neutronoptical setup depicted in Figure 2. The projective measurement of $O_{A}$ (apparatus A1, light red in Figure 2) consists of two sequential steps: first the initially prepared state is projected onto the eigenstates of $O_{A}$ by DC-2, which rotates the respective spin component of $\vec{\sigma}_{a}$ belonging to $O_{A}$ in $+z$ direction. Then, in order to complete the projective measurement the spin, which is pointing in $+z$ after the analyzer, has to be prepared in an eigenstate of $O_{A}$. This is achieved by proper positioning and magnetic field of DC-3 (thereby applying the same procedure as that for DC-1 in the initial state preparation). Finally the $B$ measurement is performed (apparatus A2, green in Figure 2) utilizing DC-4 and the second analyzer. Unlike the $O_{A}$-measurement, subsequent preparation of the eigenstates of $B$ is not necessary since the detector is insensitive to the spin state. For the measurement of the standard deviations of the observables $A$ and $B$, which are also required to test Ozawa's relation (see (5)), the two measurement apparatuses are used individually.

\section{Results and Discussion}

The experiment was carried out at the polarimeter beam line of the tangential beam port of the research reactor facility TRIGA Mark II at the Atominstitut, Vienna University of Technology, where mainly fundamental aspects of quantum mechanics are investigated [22-25]. The experimental settings for initial state $|\psi\rangle=|\Uparrow\rangle_{z} \equiv|+z\rangle$ and observables $A=\sigma_{x}$ and $B=\sigma_{y}$ require the auxiliary input states $|A \psi\rangle=$ $|B \psi\rangle=|\Downarrow\rangle_{z} \equiv|-z\rangle,|(A+\mathbb{1}) \psi\rangle=|\Uparrow\rangle_{z}+|\Downarrow\rangle_{z} \equiv|+x\rangle$, and finally $|(B+\mathbb{1}) \psi\rangle=|\Uparrow\rangle_{z}+i|\Downarrow\rangle \equiv|+y\rangle$ to be prepared. In Figure 4, explicit examples of related intensity sets for different values of $\phi$ are depicted. Standard deviations yield $\Delta(A)=\Delta(B)=1$ and the right-hand side of the uncertainty relations gives a lower bound of $(1 / 2)\langle\psi|[A, B]| \psi\rangle=1$.

In a first experimental run, $O_{A}$ is varied along the equator $(\theta=\pi / 2)$ parameterized by its azimuthal angle $\phi$. The theory curves for $\epsilon(A)$ and $\eta(B)$ are then given by

$$
\epsilon(A)=2 \sin \frac{\phi}{2}, \quad \eta(B)=\sqrt{2}|\cos \phi| .
$$
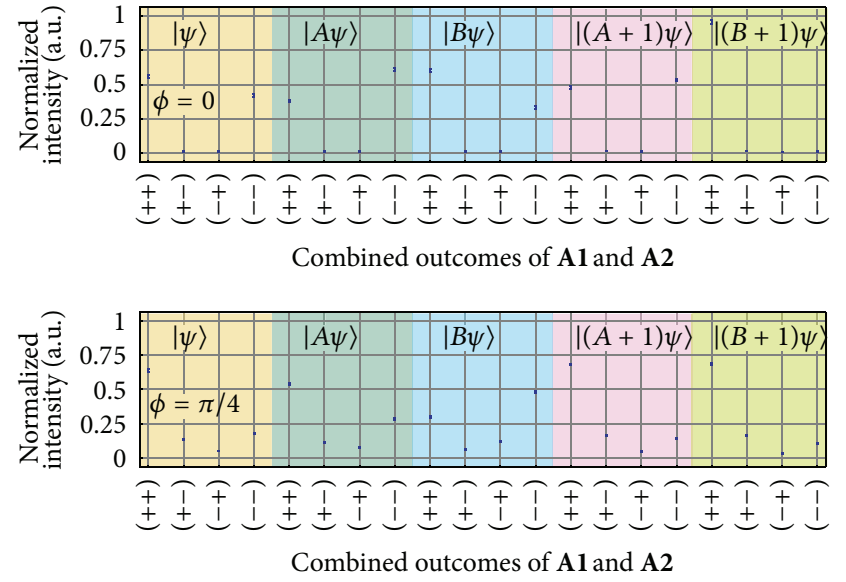

FIgURE 4: Normalized intensity of the successive measurements carried out by apparatuses $\mathbf{A 1}$ and $\mathbf{A 2}$. The combined projective measurements of $O_{A}$ and $B$ have four outcomes, denoted by $(++)$, $(+-),(-+)$, and $(--)$, and have to be recorded for each initial state, that is, $|\psi\rangle,|A \psi\rangle,|B \psi\rangle,|(A+\mathbb{1}) \psi\rangle$, and $|(B+\mathbb{1}) \psi\rangle$. Here $O_{A}$ is varied within the $x y$-plane with azimuthal angle given by $\phi=0, \pi / 4$. Error bars represent \pm one standard deviation of the normalized intensities. Some error bars are at the size of the markers.

For $\phi=0$, the error $\epsilon(A)$ vanishes and the disturbance $\eta(B)$ is maximal. The disturbance $\eta(B)$ vanishes for $O_{A}=B(\phi=$ $\pi / 2$ ) and reaches a second maximum for $O_{A}=-A$. Note that at this point also the error $\epsilon(A)$ has its (only) maximum. The famous trade-off relation, that is, the reciprocal relation for error and disturbance, only holds for $-\pi / 2 \leq \phi \leq \pi / 2$, which can be seen in Figure 5(a). The product of error and disturbance $\epsilon(A) \eta(B)$-left-hand side of (4) or Heisenberg term-is below the limit given by $(1 / 2)\langle\psi|[A, B]| \psi\rangle$ in a wide range of $\phi$-values, thereby revealing a violation of the generalized Heisenberg relation (see (4)). On the contrary, the left-hand side of Ozawa's relation $\epsilon(A) \eta(B)+\epsilon(A) \Delta(B)+$ $\Delta(A) \eta(B)$ (see (5)) is always above the lower bound defined by the expectation value of the commutator demonstrating the validity of Ozawa's new relation.

In the following experimental setting, $O_{A}$ is rotated out of the equatorial plane, when the evolution is on circles of latitude on the Bloch sphere (fixed polar angle $\theta$ ), which yields $\epsilon(A)=\sqrt{2-2 \cos \phi \sin \theta}$ and $\eta(B)=\sqrt{2-2 \sin ^{2} \phi \sin ^{2} \theta}$.

The observed values are depicted in Figure 5(b). Now neither the error $\epsilon(A)$ nor the disturbance $\eta(B)$ vanishes, since they never coincide with $A, B$, or $-B$. This behaviour affects the curves in such a way that they are now shrunken from below. The smaller $\theta$ is, the less regions the polar angle of $O_{A}$ gets where the Heisenberg term $\epsilon(A) \eta(B)$ remains below the limit. Ozawa's inequality is again fulfilled over the entire range of $\phi$. The relations shown in Figure 5 are verified for all directions of $O A$.

A modification of the measurement apparatus allows for reducing the disturbance and saturating Branciard's EDR given in (6). If we apply an arbitrary unitary rotation after the first measurement, the error remains unchained but the disturbance is altered. By investigating all possible rotation 


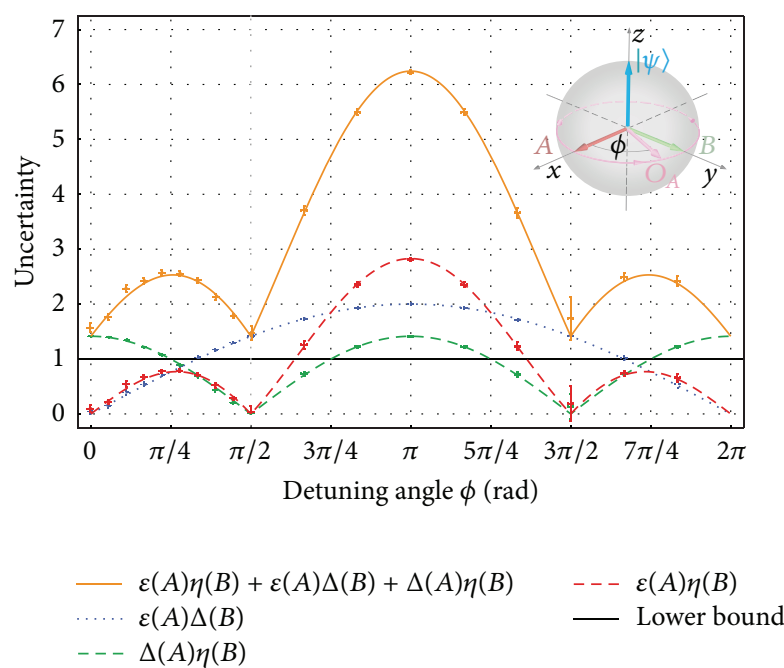

(a)

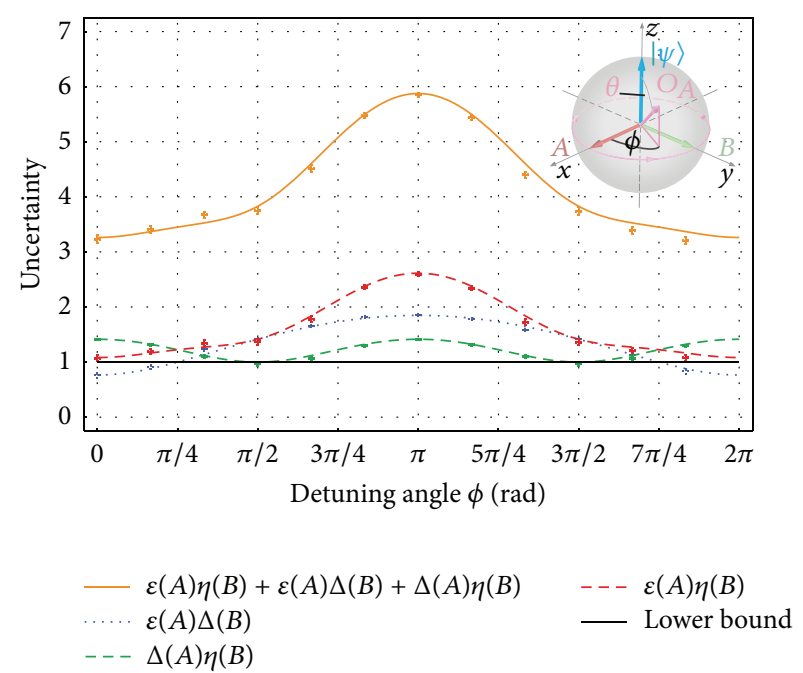

(b)

Figure 5: Experimentally determined values of $\epsilon(A) \Delta(B), \Delta(A) \eta(B)$, and $\epsilon(A) \eta(B)$. This last term corresponds to the left-hand side of the Heisenberg relation (4), and the sum of the three terms corresponds to the left-hand side of Ozawa's relation (5), including Bloch sphere representation of observables and initial state.

axes and angles, one finds out that $U_{R}=e^{-i\left(\left(\phi_{A B}-\phi\right) / 2\right) \sigma_{z}}$ minimises the disturbance yielding

$$
\epsilon(A)=2 \sin \frac{\phi}{2}, \quad \eta(B)=2 \sin \frac{\phi_{A B}-\phi}{2},
$$

where $\phi_{A B}$ is the relative angle between the $A$ and $B$ measurement direction in the equatorial plane. Note that this particular rotation just generates the eigenstates of observable $B$, that is, $| \pm y\rangle$, making the result of the optimisation procedure more intuitive. For a detailed calculation see [15].

This is experimentally achieved by an appropriate displacement of DC-3, such that the required rotation is induced, and by additional Larmor precision in the guide field. The results, both for modified and for original apparatuses, are plotted in Figure 6, demonstrating the tightness of Branciard's inequality, defined in (6).

\section{Conclusions}

To summarize, we have experimentally tested the Ozawa and Branciard error-disturbance uncertainty relations in successive neutron-spin measurements. Our experimental results clearly demonstrated the validity of Ozawa and Branciard EDRs and that the original Heisenberg EDR is violated throughout a wide range of experimental parameters.

\section{Conflict of Interests}

The authors declare that there is no conflict of interests regarding the publication of this paper.

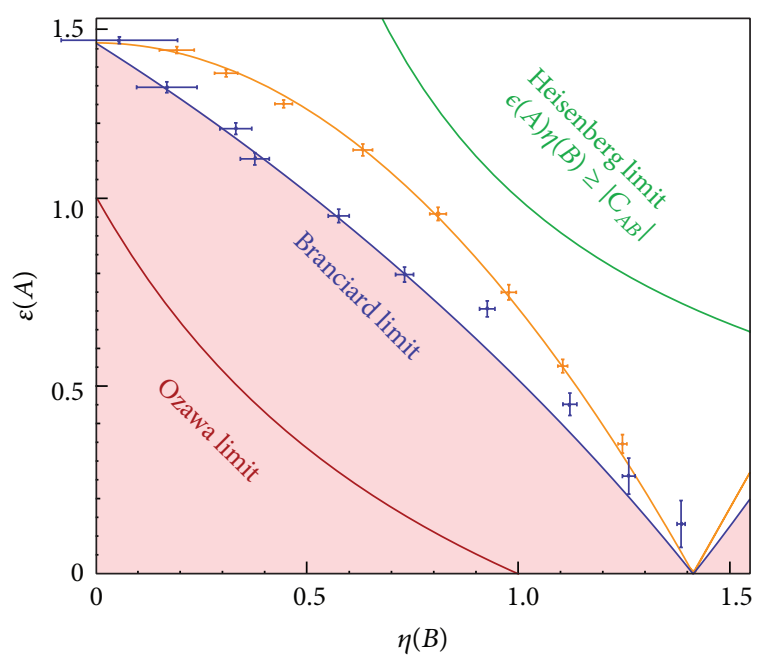

Figure 6: Results of error and disturbance plotted as $\epsilon_{A}$ versus $\eta(B)$. Blue curve: the Branciard bound as defined in (6). Blue marker: experimental results using the modified apparatus for $\epsilon(A)$ and $\eta(B)$, as defined in (10). Orange curve error and disturbance as in (9). Green curve: bound imposed by Heisenberg's original error-disturbance relation $\epsilon(A) \eta(B) \geq\left|C_{A B}\right|$, which is violated by our experimental results. Red curve: Ozawa's relation (5), which is indeed satisfied but is not saturated.

\section{Acknowledgments}

The authors thank M. Ozawa for valuable discussion. This work has been supported by the Austrian Science Fund (FWF), Projects nos. P25795-N02 and P24973-N20. 


\section{References}

[1] W. Heisenberg, "Über den anschaulichen inhalt der quantentheoretischen kinematik und mechanik," Zeitschrift für Physik, vol. 43, pp. 172-198, 1927.

[2] W. Heisenberg, The Physical Principles of Quantum Mechanics, University of Chicago Press, Chicago, Ill, USA, 1930.

[3] E. H. Kennard, "Zur Quantenmechanik einfacher Bewegungstypen," Zeitschrift für Physik, vol. 44, no. 4-5, pp. 326-352, 1927.

[4] C. G. Shull, "Single-slit diffraction of neutrons," Physical Review, vol. 179, no. 3, pp. 752-754, 1969.

[5] H. P. Yuen, "Two-photon coherent states of the radiation field," Physical Review A, vol. 13, p. 2226, 1976.

[6] G. Breitenbach, S. Schiller, and J. Mlynek, "Measurement of the quantum states of squeezed light," Nature, vol. 387, no. 6632, pp. 471-475, 1997.

[7] E. Arthurs and M. S. Goodman, "Quantum correlations: a generalized Heisenberg uncertainty relation," Physical Review Letters, vol. 60, no. 24, pp. 2447-2449, 1988.

[8] S. Ishikawa, "Uncertainty relations in simultaneous measurements for arbitrary observables," Reports on Mathematical Physics, vol. 29, no. 3, pp. 257-273, 1991.

[9] M. Ozawa, Quantum Aspects of Optical Communications, vol. 378 of Lecture Notes in Physics, Springer, 1991.

[10] J. Erhart, S. Sponar, G. Sulyok, G. Badurek, M. Ozawa, and Y. Hasegawa, "Experimental demonstration of a universally valid error-disturbance uncertainty relation in spin measurements," Nature Physics, vol. 8, pp. 185-189, 2012.

[11] G. Sulyok, S. Sponar, J. Erhart, G. Badurek, M. Ozawa, and Y. Hasegawa, "Violation of Heisenberg's error-disturbance uncertainty relation in neutron-spin measurements," Physical Review A, vol. 88, Article ID 022110, 2013.

[12] L. A. Rozema, A. Darabi, D. H. Mahler, A. Hayat, Y. Soudagar, and A. M. Steinberg, "Violation of Heisenberg's measurementdisturbance relationship by weak measurements," Physical Review Letters, vol. 109, Article ID 100404, 2012.

[13] M. M. Weston, M. J. W. Hall, M. S. Palsson, H. M. Wiseman, and G. J. Pryde, "Experimental test of universal complementarity relations," Physical Review Letters, vol. 110, no. 22, Article ID 220402, 2013.

[14] S.-Y. Baek, F. Kaneda, M. Ozawa, and K. Edamatsu, "Experimental violation and reformulation of the Heisenberg's errordisturbance uncertainty relation," Scientific Reports, vol. 3, article 2221, 2013.

[15] C. Branciard, "Error-tradeoff and error-disturbance relations for incompatible quantum measurements," Proceedings of the National Academy of Sciences of the United States of America, vol. 110, no. 17, pp. 6742-6747, 2013.

[16] M. Ringbauer, D. N. Biggerstaff, M. A. Broome, A. Fedrizzi, C. Branciard, and A. G. White, "Experimental joint quantum measurements with minimum uncertainty," Physical Review Letters, vol. 112, Article ID 020401, 2014.

[17] F. Kaneda, S. Y. Baek, M. Ozawa, and K. Edamatsu, "Experimental test of error-disturbance uncertainty relations by weak measurement," Physical Review Letters, vol. 112, Article ID 020402, 2014.

[18] M. Ozawa, "Universal uncertainty principle in the measurement operator formalism," Journal of Optics B: Quantum and Semiclassical Optics, vol. 7, article S672, 2005.

[19] R. F. Werner, "The uncertainty relation for joint measurement of postion and momentum," Quantum Information and Computation, vol. 4, no. 6, pp. 546-562, 2004.
[20] K. Koshino and A. Shimizu, "Quantum Zeno effect by general measurements," Physics Reports A: Review Section of Physics Letters, vol. 412, no. 4, pp. 191-275, 2005.

[21] M. Ozawa, "Uncertainty relations for noise and disturbance in generalized quantum measurements," Annals of Physics, vol. 311, no. 2, pp. 350-416, 2004.

[22] Y. Hasegawa and G. Badurek, "Noncommuting spinor rotation due to balanced geometrical and dynamical phases," Physical Review A, vol. 59, article 4614, 1999.

[23] J. Klepp, S. Sponar, S. Filipp, M. Lettner, G. Badurek, and Y. Hasegawa, "Observation of nonadditive mixed-state phases with polarized neutrons," Physical Review Letters, vol. 101, no. 15, Article ID 150404, 2008.

[24] S. Sponar, J. Klepp, C. Zeiner, G. Badurek, and Y. Hasegawa, "Violation of a Bell-like inequality for spin-energy entanglement in neutron polarimetry," Physics Letters A: General, Atomic and Solid State Physics, vol. 374, no. 3, pp. 431-434, 2010.

[25] S. Sponar, J. Klepp, K. Durstberger-Rennhofer et al., "Highefficiency manipulations of triply entangled states in neutron polarimetry," The New Journal of Physics, vol. 14, Article ID 053032, 2012. 

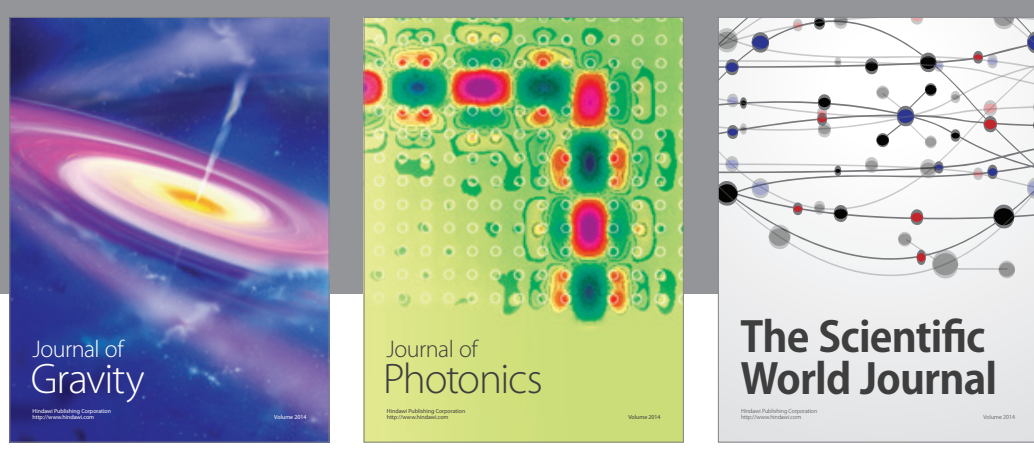

The Scientific World Journal
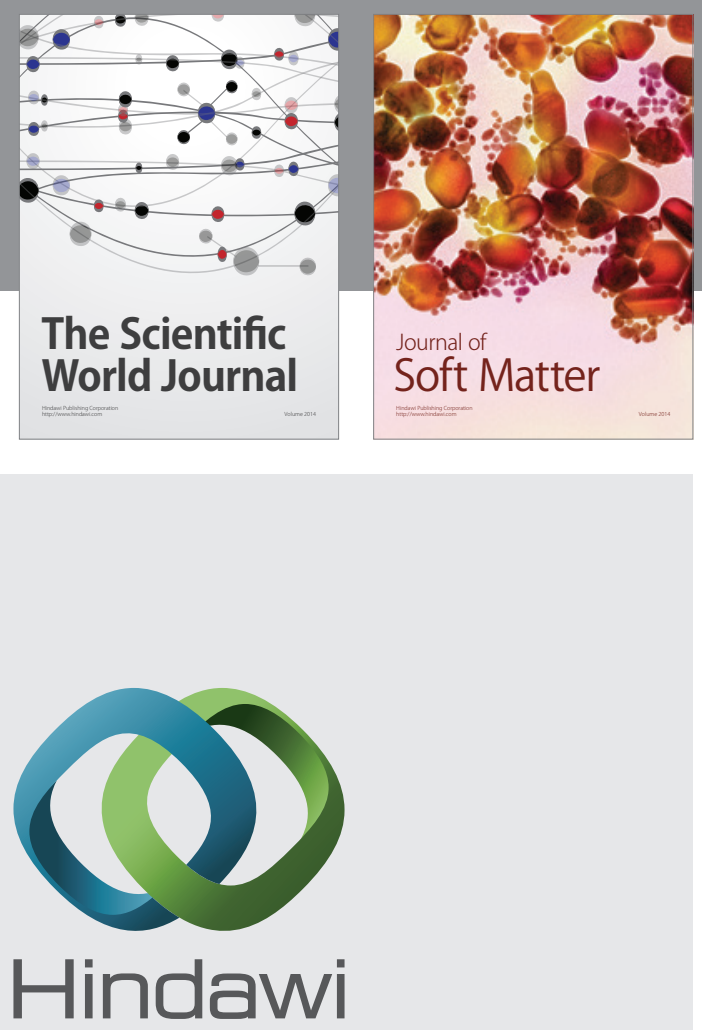

Submit your manuscripts at

http://www.hindawi.com

nternational Journal of

Statistical Mechanics
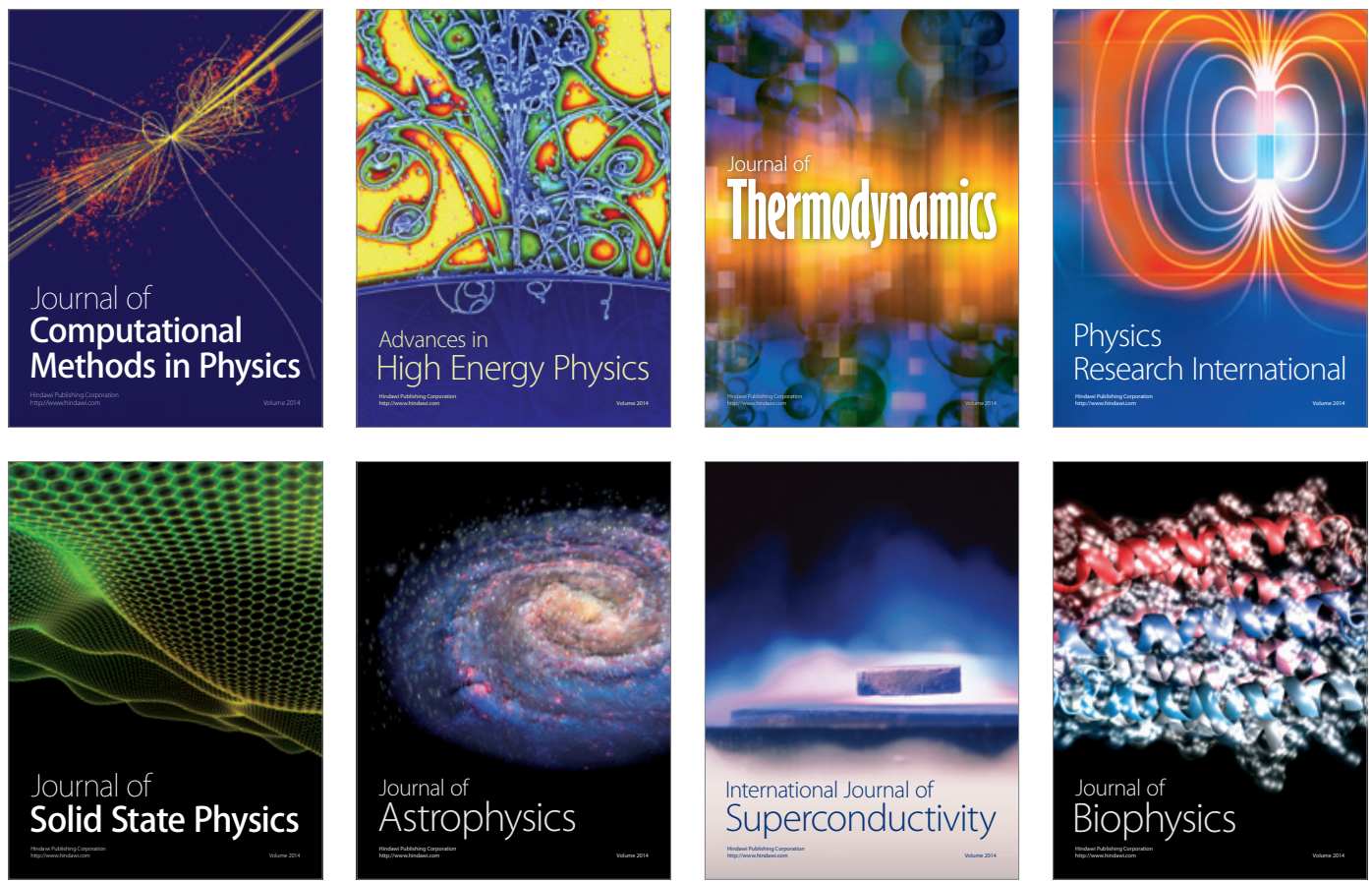
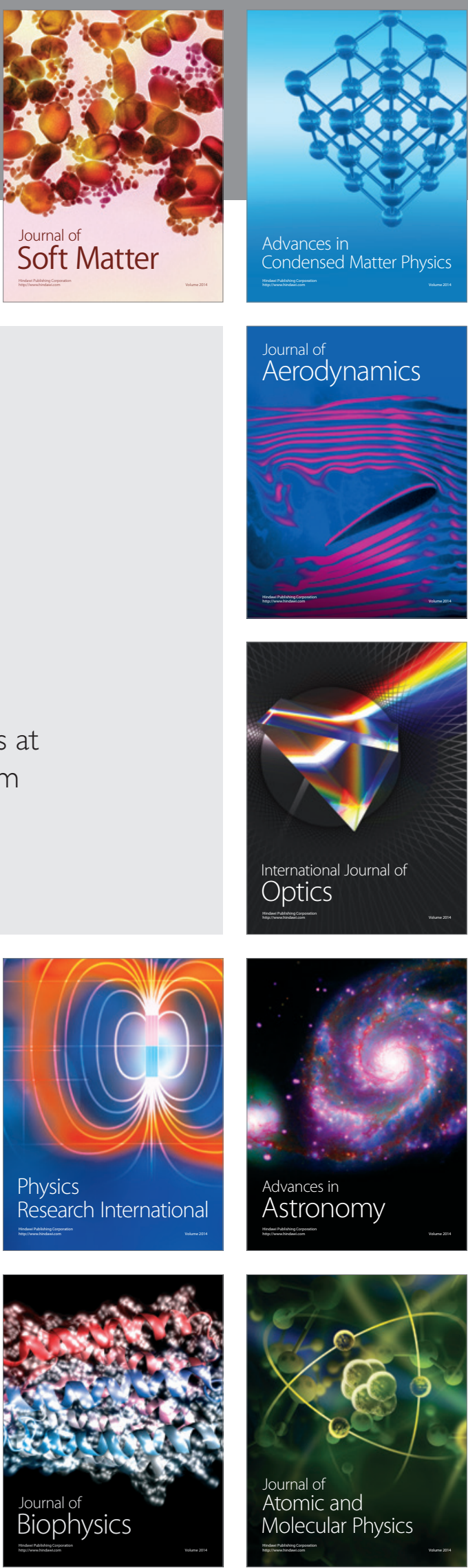